\title{
INVERTIBILITY OF LINEAR RELATIONS GENERATED BY INTEGRAL EQUATION WITH OPERATOR MEASURES
}

\author{
V.M. BRUK
}

\begin{abstract}
We investigate linear relations generated by an integral equation with operator measures on a segment in the infinite-dimensional case. In terms of boundary values, we obtain necessary and sufficient conditions under which these relations $S$ possess the properties: $S$ is a closed relation; $S$ is an invertible relation; the kernel of $S$ is finite-dimensional; the range of $S$ is closed; $S$ is a continuously invertible relation and others. The results are applied to a system of integral equations becoming a quasi-differential equation whenever the operator measures are absolutely continuous as well as to an integral equation with multi-valued impulse action.
\end{abstract}

Keywords: integral equation, operator measure, Hilbert space, linear relation, spectrum, quasi-derivative, impulse action.

Mathematics Subject Classification: 47A06, 47A10, 34B27

\section{INTRODUCTION}

Integral equations with operator measures are rather general. For instance, they cover integral-differential equations with Stiltjes integral [1, differential equations with the coefficients being generalized functions [2] (the way for reducing an integral equation to the equation in [2] was provided in [3]).

In the present work, on the segment $[a, b]$ we consider the integral equation

$$
y(t)=y_{0}-i J \int_{t_{0}}^{t}(d \mathbf{p}) y(s)-i J \int_{t_{0}}^{t}(d \mathbf{m}) f(s),
$$

where $\int_{t_{0}}^{t}$ stands for $\int_{\left[t_{0}, t\right)}$, if $t_{0}<t$; for $-\int_{\left[t, t_{0}\right)}$ if $t_{0}>t$; and for 0 if $t_{0}=t$. Here $\mathbf{p}, \mathbf{m}$ are operator-valued measures defined on Borel sets $\Delta \subset[a, b]$ and taking values in the set of linear bounded operators acting in a separable Hilbert space $H$, at that, measure $\mathbf{m}$ is non-negative (these measures are extended to a segment $\left[a_{0}, b_{0}\right] \supset\left(a_{0}, b_{0}\right) \supset[a, b]$ in the way indicated in what follows); $J$ is an operator in $H$ with the properties: $J^{*}=J, J^{2}=E$ ( $E$ indicates the identical mapping), $y_{0} \in H ; y$ is an unknown function, $f \in \mathfrak{H}=L_{2}(H, d \mathbf{m} ; a, b)$ ( $\mathfrak{H}$ is defined below). Measures $\mathbf{p}, \mathbf{m}$ are assumed to have a bounded variation in $[a, b]$.

We note that the case of an infinite dimensional $H$ differs essentially from the finitedimensional one. It is explained by the fact that the space $\mathfrak{H}=L_{2}(H, d \mathbf{m} ; a, b)$ has a rather complicated structure. The elements of this space are not necessary functions with values in $H$.

In general, equation (1) together with boundary conditions generates not linear operators but linear relations (multi-valued operators). If the boundary conditions are zero, the associated

V.M. BRUK, INVERTIBILITY OF LINEAR RELATIONS GENERATED BY INTEGRAL EQUATION WITH OPERATOR MEASURES.

(C) BRUK V.M. 2014.

The work is supported by RFBR (grant no. 13-01-00378).

Submitted May 15, 2014. 
relation is called minimal, while in the absence of boundary conditions it is called maximal. Each linear relation being a restriction of a maximal relation $L$ and an extension of a minimal relation $L_{0}$ can be defined by means of a linear relation $\theta$ involved in the boundary conditions. At that, there is a one-to-one correspondence between such relations $\theta$ and the generated relations $L_{\theta}$, $L_{0} \subset L_{\theta} \subset L$. In connection with this fact the problem appears: to select boundary conditions (i.e., relations $\theta$ ) determining relations $L_{\theta}$ with prescribed properties.

In the present work we consider properties (called states) in works [4], [5] and it is found that relation $L_{\theta}$ possesses an appropriate property if and only if the same is true for relation $\theta$. Among these properties are invertibility, continuous invertibility, Fredholm property and others. The proofs are based on the statements on abstract spaces of boundary conditions in works [6], [7].

As an application, we consider a system of integral equations and in the case of absolutely continuous measures this system becomes a quasi-differential equation with quasi-derivatives in the sense of works [8], 9]. In the last section we study an integral equation with an impulse action. Such equations describe the behavior of evolution processes involved by short-time perturbations. A mathematical model of such processes is provided in the monograph [10, Ch. 1, Sect. 1]. In the present work the impulse action is defined by a linear relation, i.e., the action is multi-valued. In work [11] there were considered differential operators generated by a strongly continuous family of evolution operators in Banach space and necessary and there were established sufficient conditions for continuous invertibility and Fredholm property of such differential operators with multi-valued impulse actions . Up to minor changes, the approach in this work is applicable to the operators considered in [11].

We note that linear relations were first employed in work [12] for the description of extensions of differential operators in terms of boundary conditions.

\section{Auxiliary STATEMENTS}

Let $H$ be a separable Hilbert space with scalar product $(\cdot, \cdot)$ and norm $\|\cdot\|$. We consider a function $\Delta \rightarrow \mathbf{P}(\Delta)$ defined on Borel sets $\Delta \subset[a, b]$ and taking values in the set of bounded linear operators acting in $H$. Function $\mathbf{P}$ is called an operator-valued measure on $[a, b]$ (see, for instance, [13, Ch. 5, Sect. 1]) if $\mathbf{P}$ vanishes on the empty set and for all non-intersecting Borel sets $\Delta_{n}$ the identity

$$
\mathbf{P}\left(\bigcup_{n=1}^{\infty} \Delta_{n}\right)=\sum_{n=1}^{\infty} \mathbf{P}\left(\Delta_{n}\right)
$$

holds true, where the series converges in the weak operator topology. By $\mathbf{V}_{\Delta}(\mathbf{P})$ we denote

$$
\mathbf{V}_{\Delta}(\mathbf{P})=\rho(\Delta)=\sup \sum_{j}\left\|\mathbf{P}\left(\Delta_{j}\right)\right\|
$$

where sup is taken over finite sums of non-intersecting Borel sets $\Delta_{j} \subset \Delta$. The number $\mathbf{V}_{\Delta}(\mathbf{P})$ is called the variation of measure $\mathbf{P}$ on Borel set $\Delta$.

Let measure $\mathbf{P}$ have a bounded variation in $[a, b]$. Then for $\rho$-almost each $\xi \in[a, b]$ there exists an operator-valued function $\xi \rightarrow \Psi(\xi)$ with values in the set of linear bounded operators in $H,\|\Psi(\xi)\|=1$, such that for each Borel set $\Delta \subset[a, b]$ the identity

$$
\mathbf{P}(\Delta)=\int_{\Delta} \Psi(\xi) d \rho
$$

holds true. Function $\Psi$ is uniquely determined up to values on a set of zero $\rho$-measure. Integral (2) converges in the sense of usual operator norm ([13, Ch. 5, Thm. 1.2]). It follows from (2) that bounded functions with values in $H$ measurable in the Borel sense are integrable w.r.t. measure $\mathbf{P}$. 
Given a measure $\mathbf{P}$ with a bounded variation, we extend it on the segment $\left[a_{0}, b_{0}\right] \supset\left(a_{0}, b_{0}\right) \supset$ $[a, b]$ letting $\mathbf{P}(\Delta)=0$ for each Borel sets $\Delta \subset\left[a_{0}, b_{0}\right] \backslash[a, b]$.

We introduce the quasi-scalar product

$$
(x, y)_{\mathbf{m}}=\int_{a_{0}}^{b_{0}}((d \mathbf{m}) x(t), y(t))
$$

on the set of step-like functions with values in $H$ defined on the segment $\left[a_{0}, b_{0}\right]$. Identifying with zero functions $y$ obeying $(y, y)_{\mathbf{m}}=0$ and making the completion, we arrive at the Hilbert space denoted by $\mathfrak{H}=L_{2}(H, d \mathbf{m} ; a, b)$. The elements of $\mathfrak{H}$ are the classes of functions identified w.r.t. the norm $\|y\|_{\mathbf{m}}=(y, y)_{\mathbf{m}}^{1 / 2}$. In order not to complicate the terminology, the class of functions with a representative $y$ is indicated by the same symbol and we write $y \in \mathfrak{H}$. The identities of the functions in $\mathfrak{H}$ are understood as the identity for associated equivalence classes. The description of space $\mathfrak{H}$ is provided in [14] (see also the references therein).

Space $\mathfrak{H}$ and linear relations considered in what follows do not change once we replace interval $\left(a_{0}, b_{0}\right)$ by $\left(a_{0}^{\prime}, b_{0}^{\prime}\right)$, where points $a_{0}^{\prime}, b_{0}^{\prime}$ are introduced as points $a_{0}, b_{0}$, i.e., $\left[a_{0}^{\prime}, b_{0}^{\prime}\right] \supset\left(a_{0}^{\prime}, b_{0}^{\prime}\right) \supset$ $[a, b]$ and $\mathbf{p}(\Delta)=\mathbf{m}(\Delta)=0$ for each Borel set $\Delta \subset\left[a_{0}^{\prime}, b_{0}^{\prime}\right] \backslash[a, b]$.

We consider equations

$$
\begin{aligned}
& y(t)=y_{0}-i J \int_{t_{0}}^{t}(d \mathbf{p}) y(s)-i \lambda J \int_{t_{0}}^{t}(d \mathbf{m}) y(s)-i J \int_{t_{0}}^{t}(d \mathbf{m}) f(s), \\
& z(t)=z_{0}-i J \int_{t_{0}}^{t}\left(d \mathbf{p}^{*}\right) z(s)-i \bar{\lambda} J \int_{t_{0}}^{t}(d \mathbf{m}) z(s)-i J \int_{t_{0}}^{t}(d \mathbf{m}) g(s),
\end{aligned}
$$

where $y_{0}, z_{0} \in H, f, g \in \mathfrak{H}, \lambda \in \mathbb{C}, t, t_{0} \in\left[a_{0}, b_{0}\right]$. We note that as $\lambda=0$, equation (3) becomes equation (1).

It follows from [3], [14] that for each $y_{0}, z_{0} \in H, f, g \in \mathfrak{H}, \lambda \in \mathbb{C}$ equations (3), (4) have unique solutions. These solutions are left continuous and $y_{0}=y\left(t_{0}\right), z_{0}=z\left(t_{0}\right)$. By $W(t, \lambda)$, $U(t, \bar{\lambda})$ we denote operator solutions to equations

$$
\begin{aligned}
& W(t, \lambda) x_{0}=x_{0}-i J \int_{t_{0}}^{t}(d \mathbf{p}) W(s, \lambda) x_{0}-i \lambda J \int_{t_{0}}^{t}(d \mathbf{m}) W(s, \lambda) x_{0}, \\
& U(t, \bar{\lambda}) \tilde{x}_{0}=\tilde{x}_{0}-i J \int_{t_{0}}^{t}\left(d \mathbf{p}^{*}\right) U(s, \bar{\lambda}) \tilde{x}_{0}-i \bar{\lambda} J \int_{t_{0}}^{t}(d \mathbf{m}) U(s, \bar{\lambda}) \tilde{x}_{0},
\end{aligned}
$$

where $x_{0}, \tilde{x}_{0} \in H$. It follows from [3], [14 that $U^{*}(t, \bar{\lambda}) J W(t, \lambda)=J, W(t, \lambda) J U^{*}(t, \bar{\lambda})=J$ and functions $\lambda \rightarrow W(t, \lambda), \lambda \rightarrow U(t, \lambda)$ are holomorphic in $\lambda \in \mathbb{C}$ for each fixed $t \in\left[a_{0}, b_{0}\right]$. Reproducing the proof of similar statements in [3], [14], we arrive at the following lemma.

Lemma 1. Functions $y, z$ solve equations (3), (4), respectively, if and only if $y, z$ read as

$$
\begin{aligned}
& y(t)=W(t, \lambda) y_{0}-W(t, \lambda) i J \int_{t_{0}}^{t} U^{*}(s, \bar{\lambda})(d \mathbf{m}) f(s), \\
& z(t)=U(t, \bar{\lambda}) z_{0}-U(t, \bar{\lambda}) i J \int_{t_{0}}^{t} W^{*}(s, \lambda)(d \mathbf{m}) g(s) .
\end{aligned}
$$

\section{MAXIMAL AND MiNimAL RELATIONS}

Let $\mathbf{B}_{1}, \mathbf{B}_{2}$ be Banach spaces. By a linear relation $\mathrm{T}$ we mean any linear manifold $\mathrm{T} \subset$ $\mathbf{B}_{1} \times \mathbf{B}_{2}$. The terminology on linear relations can be found, for instance, in [4], [5]. In what follows we make use of the following notations: $\{\cdot, \cdot\}$ is an ordered pair; ker $\mathrm{T}$ is the set of elements $x \in \mathbf{B}_{1}$ such that $\{x, 0\} \in \mathrm{T}$; KerT is the set of ordered pairs $\{x, 0\} \in \mathrm{T} ; \mathcal{D}(\mathrm{T})$ is the domain of $\mathrm{T}$, i.e., the set of the elements $x \in \mathbf{B}_{1}$ such that for each of them there 
exists an element $x^{\prime} \in \mathbf{B}_{2}$ such that $\left\{x, x^{\prime}\right\} \in \mathrm{T} ; \mathcal{R}(\mathrm{T})$ is the range of $\mathrm{T}$, i.e., the set of the elements $x^{\prime} \in \mathbf{B}_{2}$ such that for each of them there exists an element $x \in \mathbf{B}_{1}$ such that $\left\{x, x^{\prime}\right\} \in \mathrm{T} ; \mathrm{T}^{-1}$ is the relation inverse for $\mathrm{T}$, i.e., the relation formed by the pairs $\left\{x^{\prime}, x\right\}$, where $\left\{x, x^{\prime}\right\} \in \mathrm{T}$. Relation $\mathrm{T}$ is called surjective if $\mathcal{R}(\mathrm{T})=\mathbf{B}_{2}$. It is called invertible or injective if ker $\mathrm{T}=\{0\}$ (i.e., the relation $\mathrm{T}^{-1}$ is an operator); it is called continuously invertible if it is closed, invertible and surjective (i.e., $\mathrm{T}^{-1}$ is a bounded everywhere defined operator). The sum of relations $\mathrm{T}_{1}, \mathrm{~T}_{2} \subset \mathbf{B}_{1} \times \mathbf{B}_{2}$ is the relation $\mathrm{T}_{1}+\mathrm{T}_{2}$ consisting of pair $\left\{x, x_{1}+x_{2}\right\}$, where $x \in \mathcal{D}\left(\mathrm{T}_{1}\right) \cap \mathcal{D}\left(\mathrm{T}_{2}\right),\left\{x, x_{1}\right\} \in \mathrm{T}_{1},\left\{x, x_{2}\right\} \in \mathrm{T}_{2}$. The product of relations $\mathrm{T} \subset \mathbf{B}_{1} \times \mathbf{B}_{2}$, $\mathrm{S} \subset \mathbf{B}_{2} \times \mathbf{B}_{3}$ is the relation ST formed by the pairs $\left\{x_{1}, x_{3}\right\} \in \mathbf{B}_{1} \times \mathbf{B}_{3}$ such that for each of them there exists an element $x_{2}$ obeying $\left\{x_{1}, x_{2}\right\} \in \mathrm{T},\left\{x_{2}, x_{3}\right\} \in \mathrm{S}$.

In what follows $\rho(\mathrm{T})$ indicates the resolvent set of a closed relation T, i.e., the set of points $\lambda \in \mathbb{C}$ such that the relation $(\mathrm{T}-\lambda E)^{-1}$ is a bounded everywhere defined operator; $\sigma_{c}(\mathrm{~T})$ $\left(\sigma_{r}(\mathrm{~T})\right)$ is the continuous (residual) spectrum of relation $\mathrm{T}$, i.e., the set of points $\lambda \in \mathbb{C}$ such that relation $(\mathrm{T}-\lambda E)^{-1}$ is a densely defined and unbounded (non-densely defined) operator. Symbol $\sigma_{p}(\mathrm{~T})$ denotes the point spectrum of relation $\mathrm{T}$, i.e., the set of points $\lambda \in \mathbb{C}$ such that the relation $(\mathrm{T}-\lambda E)^{-1}$ is not an operator. Linear operators are treated as linear relations, this is why the notation $\left\{x_{1}, x_{2}\right\} \in \mathrm{T}$ is used also for operator T. Since all considered relations are linear, we shall often omit word "linear".

Let $L^{\prime}$ be the relation consisting of the pairs $\{\tilde{y}, \tilde{f}\} \in \mathfrak{H} \times \mathfrak{H}$ such that for each of them there exists a pair $\{y, f\}$ identified with $\{\tilde{y}, \tilde{f}\}$ in $\mathfrak{H} \times \mathfrak{H}$ and satisfying equation (1). By $L$ we denote the closure of $L^{\prime}$ and we call $L$ the maximal relation generated by equation (1). Generally speaking, relation $L$ is not an operator since function $y$ can happen to be identified with zero in $\mathfrak{H}$, while $f$ is non-zero. We define the minimal relation $L_{0}$ as the restriction of $L^{\prime}$ to the set of functions $y$ such that $y\left(a_{0}\right)=y\left(b_{0}\right)=0$, where $y$ is a solution to (1).

Remark 1. The definition of points $a_{0}, b_{0}$ and identities $\mathbf{p}(\Delta)=\mathbf{m}(\Delta)=0$ valid for each Borel set $\Delta \subset\left[a_{0}, b_{0}\right] \backslash[a, b]$ yield $y\left(a_{0}\right)=\lim _{t \rightarrow a-0} y(t), y\left(b_{0}\right)=\lim _{t \rightarrow b+0} y(t)$. The maximal and minimal relations do not change if we replace the interval $\left(a_{0}, b_{0}\right)$ by $\left(a_{0}^{\prime}, b_{0}^{\prime}\right)$, where points $a_{0}^{\prime}$, $b_{0}^{\prime}$ are defined in the same way as $a_{0}, b_{0}$, and measures $\mathbf{p}, \mathbf{m}$ are extended to the interval $\left(a_{0}^{\prime}, b_{0}^{\prime}\right)$ in the same way as $\left(a_{0}, b_{0}\right)$. This is why minimal relation $L_{0}$ can be defined as the restriction of $L^{\prime}$ to the set of functions y compactly supported in $\left(a_{0}, b_{0}\right)$, where $y$ solves (1).

We denote by $Q_{0}$ (by $\widehat{Q}_{0}$ ) the set of elements $x \in H$ such that as $\mu \in \mathbb{C}$, the function $t \rightarrow W(t, \mu) x\left(t \rightarrow U(t, \mu) x\right.$, respectively) is identified with zero in $\mathfrak{H}$. We let $Q=H \ominus Q_{0}$ and $\widehat{Q}=H \ominus \widehat{Q}_{0}$. Sets $Q_{0}, \widehat{Q}_{0}$ (and hence $Q, \widehat{Q}$ ) are independent of changing point $\mu$ to another point $\lambda \in \mathbb{C}$. It is implied by the identities

$$
\begin{aligned}
& W(t, \lambda) c=W(t, 0) c-\lambda W(t, 0) i J \int_{t_{0}}^{t} U^{*}(s, 0)(d \mathbf{m}) W(s, \lambda) c, \\
& W(t, 0) c=W(t, \lambda) c+\lambda W(t, \lambda) i J \int_{t_{0}}^{t} U^{*}(s, \bar{\lambda})(d \mathbf{m}) W(s, 0) c, \quad c \in H,
\end{aligned}
$$

followed by (5), (6). Similar identities for $U(t, \lambda), U(t, 0)$ are obtained from (7), (8) by replacing $W$ by $U$ and $U$ by $W$.

On linear manifolds $Q$ and $\widehat{Q}$ we introduce the norms

$$
\begin{aligned}
& \|c\|_{-}=\left(\int_{a_{0}}^{b_{0}}((d \mathbf{m}) W(s, \mu) c, W(s, \mu) c)\right)^{1 / 2}, \quad \mu \in \mathbb{C}, \quad c \in Q, \\
& \|\widehat{c}\|_{-}=\left(\int_{a_{0}}^{b_{0}}((d \mathbf{m}) U(s, \mu) \widehat{c}, U(s, \mu) \widehat{c})\right)^{1 / 2}, \quad \mu \in \mathbb{C}, \quad \widehat{c} \in \widehat{Q} .
\end{aligned}
$$


Replacing measure $\mathbf{P}$ by $\mathbf{m}$ in formula (2), we get

$$
\|c\|_{-}=\left(\int_{a_{0}}^{b_{0}}(\Psi(s) W(s, \mu) c, W(s, \mu) c) d \rho\right)^{1 / 2} \leqslant \gamma\|c\|, \quad \gamma>0, \quad c \in Q .
$$

By $Q_{-}, \widehat{Q}_{-}$we denote the completion of $Q, \widehat{Q}$ in norms $(9),(10)$, respectively. It follows from (7), (8) that replacing $\mu$ by $\lambda \in \mathbb{C}$ in (9) (or in (10) ) leads to the same set $Q_{-}\left(\widehat{Q}_{-}\right.$, respectively) with an equivalent norm. It follows from (11) and similar inequality for norm (10) that spaces $Q_{-}, \widehat{Q}_{-}$can be treated as spaces with negative norm w.r.t. $Q\left[13\right.$, Ch. 1, Sect. 1]. By $Q_{+}, \widehat{Q}_{+}$ we denote the associated spaces with positive norm. The definition of spaces with positive and negative norms imply that $Q_{+} \subset Q, \widehat{Q}_{+} \subset Q$.

Suppose that sequences $\left\{c_{n}\right\}$ and $\left\{\widehat{c}_{n}\right\}\left(c_{n} \in Q, \widehat{c}_{n} \in \widehat{Q}\right)$ converge respectively in $Q_{-}$and $\widehat{Q}_{-}$to $c_{0} \in Q_{-}$and $\widehat{c}_{0} \in \widehat{Q}_{-}$. Then sequences $\left\{W(\cdot, \lambda) c_{n}\right\},\left\{U(\cdot, \lambda) \widehat{c}_{n}\right\}$ are fundamental in $\mathfrak{H}$ and hence, they converge to some elements in $\mathfrak{H}$. By $W(\cdot, \lambda) c_{0}$ and $U(\cdot, \lambda) \widehat{c}_{0}$ we denote these elements and $\mathcal{W}(\lambda), \mathcal{U}(\lambda)$ stand for the operators $c \rightarrow W(\cdot, \lambda) c$ and $\widehat{c} \rightarrow U(\cdot, \lambda) \widehat{c}$, respectively, where $c \in Q_{-}, \widehat{c} \in \widehat{Q}_{-}$. Operators $\mathcal{W}(\lambda): Q_{-} \rightarrow \mathfrak{H}, \mathcal{U}(\lambda): \widehat{Q}_{-} \rightarrow \mathfrak{H}$ are continuous, one-to-one and their domains are closed. Thus, adjoint operators $\mathcal{W}^{*}(\lambda), \mathcal{U}^{*}(\lambda)$ map continuously $\mathfrak{H}$ on $Q_{+}, \widehat{Q}_{+}$, respectively. For each $x \in Q, f \in \mathfrak{H}$ we have

$$
(f, \mathcal{W}(\lambda) x)_{\mathbf{m}}=\int_{a_{0}}^{b_{0}}((d \mathbf{m}) f(s), W(s, \lambda) x)=\int_{a_{0}}^{b_{0}}\left(W^{*}(s, \lambda)(d \mathbf{m}) f(s), x\right)=\left(\mathcal{W}^{*}(\lambda) f, x\right) .
$$

Similar identity holds for operator $\mathcal{U}(\lambda)$. Due to the dense embedding of $Q, \widehat{Q}$ into $Q_{-}, \widehat{Q}_{-}$, respectively, we obtian

$$
\mathcal{W}^{*}(\lambda) f=\int_{a_{0}}^{b_{0}} W^{*}(s, \lambda)(d \mathbf{m}) f(s), \quad \mathcal{U}^{*}(\lambda) g=\int_{a_{0}}^{b_{0}} U^{*}(s, \lambda)(d \mathbf{m}) g(s) .
$$

Hence, we have proven the following lemma.

Lemma 2. Operators $\mathcal{W}^{*}(\lambda), \mathcal{U}^{*}(\lambda)$ map continuously $\mathfrak{H}$ onto $Q_{+}, \widehat{Q}_{+}$, respectively and are given by 12 .

The next theorem and corollaries are proven in the same way as similar statements in [3], [14], [15].

Theorem 1. A pair $\{\tilde{y}, \tilde{f}\} \in \mathfrak{H} \times \mathfrak{H}$ belongs to the relation $L-\lambda E$ if and only if there exists a pair $\{y, f\}$ identified with $\{\tilde{y}, \tilde{f}\}$ in $\mathfrak{H} \times \mathfrak{H}$ such that this pair satisfies identity (5), where $y_{0} \in Q_{-}, f \in \mathfrak{H}$.

Corollary 1. Relation $L_{0}$ is closed.

Corollary 2. The range of the relation $L_{0}-\lambda E$ consists of the elements $f \in \mathfrak{H}$ satisfying the identity

$$
\mathcal{U}^{*}(\bar{\lambda}) f=\int_{a_{0}}^{b_{0}} U^{*}(s, \bar{\lambda})(d \mathbf{m}) f(s)=0 .
$$

Corollary 3. Operator $\mathcal{W}(\lambda)$ is a continuous one-to-one mapping of $Q_{-}$onto $\operatorname{ker}(L-\lambda E)$.

\section{Spaces of Boundary VAlues and states of Linear RElations}

In what follows we shall make use of space of boundary values (SBV) for the relation $L-\lambda E$. Let $\mathbf{B}_{1}, \mathbf{B}_{2}, B_{1}, B_{2}$ be Banach spaces, $T \subset \mathbf{B}_{1} \times \mathbf{B}_{2}$ be a closed linear relation $\delta: T \rightarrow B_{1} \times B_{2}$ be a linear operator, $\delta_{j}=P_{j} \delta, j=1,2\left(P_{j}\right.$ indicates the natural projection on set $G_{j}$ in the Cartesian product $\left.G=G_{1} \times G_{2}\right)$. A quadruple $\left(B_{1}, B_{2}, \delta_{1}, \delta_{2}\right)$ is called SBV for relation 
$T$ (cf. [6], [7] and the references therein), if $\delta$ maps continuously $T$ onto $B_{1} \times B_{2}$ and the restriction of $\delta_{1}$ onto Ker $T$ is a one-to-one mapping of KerT onto $B_{1}$. We define an operator $\Phi_{\delta}: B_{1} \rightarrow B_{2}$ and a relation $T_{0}$ by the identities $\Phi_{\delta}=\delta_{2}\left(\left.\delta_{1}\right|_{\operatorname{Ker} \mathrm{T}}\right)^{-1}, T_{0}=\operatorname{ker} \delta$. We note that operator $\Phi_{\delta}$ is bounded. It follows from the definition of SBV that there exists a one-to-one correspondence between relations $\widehat{T}$ with the property $T_{0} \subset \widehat{T} \subset T$ and relations $\theta \subset B_{1} \times B_{2}$ and this correspondence is determined by identity $\delta \widehat{T}=\theta$. In this case we denote $\widehat{T}=T_{\theta}$. Similar notations are also used below.

Let $S$ be a linear relation, $S \subset B_{1}^{\prime} \times B_{2}^{\prime}$, where $B_{1}^{\prime}, B_{2}^{\prime}$ are Banach spaces. The following conditions are borrowed from [4], [5]: 1) $S$ is closed; 2) $\operatorname{ker} S=\{0\} ; 3) \operatorname{dim} \operatorname{ker} S<\infty$; 4) relation $S$ is well-defined; 5) $\overline{\mathcal{R}(S)}=\mathcal{R}(S)$; 6) $\mathcal{R}(S)$ is a closed subspace in $B_{2}^{\prime}$ of finite codimension; 7) $\mathcal{R}(S)=B_{2}^{\prime}$; 8) $S$ is continuously invertible.

Following [4], [5], we shall say that relation $S$ is in state $k$ if it satisfies condition $k$ ). Condition 4) means the invertibility of relation $S$ and the closeness of range of $\mathcal{R}(S)$ [5]. Relation $S$ is called Fredholm if it satisfies Conditions 3), 6).

Theorem 2. Let $\mathcal{R}(T)=\mathbf{B}_{2}$. Relation $T_{\theta}$ is in state $k(1 \leqslant k \leqslant 8)$ if and only if the same is true for the relation $\theta-\Phi_{\delta}$.

The proof is implied by the following lemma established in [7].

Lemma 3. Relation $T_{\theta}$ is closed if and only if the same is true for relation $\theta$. Let $\mathcal{R}(T)=\mathbf{B}_{2}$. The following statements hold true:

1) the range of $\mathcal{R}\left(T_{\theta}\right)$ is closed if and only if the range of $\mathcal{R}\left(\theta-\Phi_{\delta}\right)$ is closed;

2) $\operatorname{dim} \mathbf{B}_{2} / \overline{\mathcal{R}\left(T_{\theta}\right)}=\operatorname{dim} B_{2} / \overline{\mathcal{R}\left(\theta-\Phi_{\delta}\right)}$;

3) $\operatorname{dim} \operatorname{ker} T_{\theta}=\operatorname{dim} \operatorname{ker}\left(\theta-\Phi_{\delta}\right)$.

Let us construct the space of boundary values for relation $L$. We denote $Q_{b}=W\left(b_{0}, 0\right) J \widehat{Q}_{+}$. Operator $W\left(b_{0}, 0\right)$ is a one-to-one mapping of $H$ onto $H$. Employing the latter identity, in $Q_{b}$ we introduce the norm of space $Q_{+}$. Without loss of generality we can assume that $t_{0}=a_{0}$, $W\left(a_{0}, \lambda\right)=E$.

In accordance with Theorem 1, a pair $\{\tilde{y}, \tilde{f}\} \in \mathfrak{H} \times \mathfrak{H}$ belongs to the relation $L-\lambda E$ if and only if there exists a pair $\{y, f\}$ identified with $\{\tilde{y}, \tilde{f}\}$ in $\mathfrak{H} \times \mathfrak{H}$ and satisfying

$$
y(t)=W(t, \lambda) c_{\lambda}+F_{\lambda}(t)
$$

where $c_{\lambda} \in Q_{-}$,

$$
F_{\lambda}(t)=-W(t, \lambda) i J \int_{a_{0}}^{t} U^{*}(s, \bar{\lambda})(d \mathbf{m}) f(s) d s .
$$

With each pair $\{y, f\}$ represented by (13) as $\lambda=0$ we associated a pair of boundary values

$$
\mathrm{Y}=\tilde{\delta}_{1}\{y, f\}=c_{0} \in Q_{-}, \quad \mathrm{Y}^{\prime}=\tilde{\delta}_{2}\{y, f\}=-W\left(b_{0}, 0\right) J \int_{a_{0}}^{b_{0}} U^{*}(s, 0)(d \mathbf{m}) f(s) d s \in Q_{b} .
$$

It follows from (13), (14) that if pairs $\{y, f\},\{\tilde{y}, \tilde{f}\} \in L$ are identified in $\mathfrak{H} \times \mathfrak{H}$, their boundary values coincide.

We note that if $c_{0} \in Q$ (i.e., $\{y, f\} \in L^{\prime}$ ), then

$$
\mathrm{Y}=y\left(a_{0}\right), \quad \mathrm{Y}^{\prime}=y\left(b_{0}\right)-W\left(b_{0}, 0\right) y\left(a_{0}\right) .
$$

We let $\tilde{\delta}\{y, f\}=\left\{\mathrm{Y}, \mathrm{Y}^{\prime}\right\}$. Theorem 1, Lemma 2, and Corollary 3 imply that a quadruple $\left(Q_{-}, Q_{b}, \tilde{\delta}_{1}, \tilde{\delta}_{2}\right)$ is a SBV for relation $L$; at that, $\operatorname{ker} \tilde{\delta}=L_{0}$. As above, $L_{\theta}$ is a linear relation such that $L_{0} \subset L_{\theta} \subset L$ and $\tilde{\delta} L_{\theta}=\theta \subset Q_{-} \times Q_{b}$. 
Let $\{y, f\} \in L$. Then $\{y, f-\lambda y\} \in L-\lambda E$. We let $\delta(\lambda)\{y, f-\lambda y\}=\tilde{\delta}\{y, f\}$ and $\delta_{j}(\lambda)=P_{j} \delta(\lambda)$, where $P_{1}, P_{2}$ are natural projections of $Q_{-} \times Q_{b}$ on $Q_{-}, Q_{b}$, respectively. It is clear that $\tilde{\delta}=\delta(0)$.

Operator $\tilde{\delta}$ maps continuously $L$ onto $Q_{-} \times Q_{b}$, while operator mapping each pair $\{y, f\} \in L$ into pair $\{y, f-\lambda y\} \in L-\lambda E$ is continuous and a one-to-one correspondence between $L$ and $L-\lambda E$. Hence, operator $\delta(\lambda)$ maps continuously $L-\lambda E$ onto $Q_{-} \times Q_{b}$. It follows from (7), (8) that the restriction of $\delta_{1}(\lambda)$ to $\operatorname{Ker}(L-\lambda E)$ is a one-to-one correspondence between $\operatorname{Ker}(L-\lambda E)$ and $Q_{-}$. Thus, for each $\lambda \in \mathbb{C}$, a quadruple $\left(Q_{-}, Q_{b}, \delta_{1}(\lambda), \delta_{2}(\lambda)\right)$ is SBV for the relation $L-\lambda E$. The operator $\Phi_{\delta(\lambda)}=\delta_{2}(\lambda)\left(\left.\delta_{1}(\lambda)\right|_{\operatorname{Ker}(L-\lambda E)}\right)^{-1}$ reads as

$$
\Phi_{\delta(\lambda)}=-\lambda W\left(b_{0}, 0\right) J \int_{a_{0}}^{b_{0}} U^{*}(s, 0)(d \mathbf{m}) W(s, \lambda) d s .
$$

If $c_{0} \in Q$, then

$$
\Phi_{\delta(\lambda)} c_{0}=\left(W\left(b_{0}, \lambda\right)-W\left(b_{0}, 0\right)\right) c_{0} .
$$

Theorem 2 implies the following statement.

Theorem 3. Relation $L_{\theta}-\lambda E$ is in state $k$ if and only if the same is true for the relation $\theta-\Phi_{\delta(\lambda)}$.

Corollary 4. Suppose that relation $\theta$ is closed. A point $\lambda$ belongs to point spectrum $\sigma_{p}\left(L_{\theta}\right)$ of relation $L_{\theta}$ if and only if $\operatorname{ker}\left(\theta-\Phi_{\delta(\lambda)}\right) \neq\{0\}$. A point $\lambda$ belongs to residual spectrum $\sigma_{r}\left(T_{\theta}\right)$ (to continuous spectrum $\sigma_{c}\left(L_{\theta}\right)$ ) if and only if relation $\left(\theta-\Phi_{\delta(\lambda)}\right)^{-1}$ if a non-densely defined (densely defined and unbounded) operator. A point $\lambda$ belongs to resolvent set $\rho\left(L_{\theta}\right)$ if and only if $\left(\theta-\Phi_{\delta(\lambda)}\right)^{-1}$ is a bounded everywhere defined operator.

In conclusion of this section we consider a system integral equations becoming quasidifferential equation in the case of absolute continuity of operator measures.

Let $\mathcal{H}$ be a finite-dimensional Hilbert space. On the segment $[a, b]$ we consider the system of $r \geqslant 2$ equations

$$
\begin{array}{r}
u_{j-1}(t)=u_{j-1}\left(t_{0}\right)+\sum_{k=1}^{j+1} \int_{t_{0}}^{t}\left(d \mathbf{p}_{j, k}\right) u_{k-1}(s), \quad j=1, \ldots, r-1, \\
u_{r-1}(t)=u_{r-1}\left(t_{0}\right)+\sum_{k=1}^{r} \int_{t_{0}}^{t}\left(d \mathbf{p}_{r, k}\right) u_{k-1}(s)+\lambda i^{-r} \int_{t_{0}}^{t}\left(d \mathbf{m}_{1}\right) u_{0}(s)+i^{-r} \int_{t_{0}}^{t}\left(d \mathbf{m}_{1}\right) f(s),
\end{array}
$$

where $\mathbf{p}_{j, k}, \mathbf{m}_{1}$ are operator measures on $[a, b]$ whose values are linear operators in $\mathcal{H}$, at that, measure $\mathbf{m}_{1}$ is non-negative; $f \in L_{2}\left(\mathcal{H}, d \mathbf{m}_{1} ; a, b\right) ; \lambda \in \mathbb{C} ; u=u_{0}, u_{1}, \ldots, u_{r-1}$ are unknown functions. Measures $\mathbf{p}_{j, k}$ are assumed to satisfy the conditions: (a) $\mathbf{p}_{j, k}=0$ as $k>j+1$; (b) there exist operator-valued functions $t \rightarrow p_{j, j+1}(t)$ with norms $t \rightarrow\left\|p_{j, j+1}(t)\right\| \in L_{1}(a, b)$ such that $\mathbf{p}_{j, j+1}(\Delta)=\int_{\Delta} p_{j, j+1}(t) d t$ for each Borel set $\Delta$ (i.e., measures $\mathbf{p}_{j, j+1}$ are absolutely continuous) and operators $p_{j, j+1}(t)$ have inverse for each $t \in[a, b]$.

Let us reduce system (17) to first order equations. We denote $\mathbf{p}=i J \mathbf{P}$, where $\mathbf{P}$ is a matrix of order $r$ with entries $\mathbf{p}_{j, k}, J=i^{r+1} \Lambda, \Lambda$ is the matrix whose secondary diagonal is $-E, E, \ldots,(-1)^{r} E$ (from up to down), while all other entries are zero, $\mathbf{m}$ is the matrix of order $r$ having $\mathbf{m}_{1}$ at the intersection of the first row and the first column, while other elements are zero. We also let $\widehat{u}=\operatorname{col}\left(u_{0}, \ldots, u_{r-1}\right), \breve{f}=\operatorname{col}(f, 0, \ldots, 0)$ (the column of length $r$ ). In column $\breve{f}$ there can be arbitrary functions instead of zeroes.

In terms of the above notations, system (17) casts into the form (3), where $y=\widehat{u}, H=\mathcal{H}^{r}$ :

$$
\widehat{u}(t)=\widehat{u}\left(t_{0}\right)-i J \int_{t_{0}}^{t}(d \mathbf{p}) \widehat{u}(s)-i \lambda J \int_{t_{0}}^{t}(d \mathbf{m}) \widehat{u}(s)-i J \int_{t_{0}}^{t}(d \mathbf{m}) \breve{f}(s) .
$$


Functions $u_{k}(k=0, \ldots, r-1)$ solving system (17) are called quasi-derivatives of function $u=u_{0}$ and we denote $u_{k}=u^{[k]}$. By (17) we obtain

$$
\int_{t_{0}}^{t} p_{j, j+1}(s) u_{j}(s) d s=u_{j-1}(t)-u_{j-1}\left(t_{0}\right)-\sum_{k=1}^{j} \int_{t_{0}}^{t}\left(d \mathbf{p}_{j, k}\right) u_{k-1}(s)
$$

for each $a_{0} \leqslant t \leqslant b_{0}, j=1, \ldots, r-1$.

The left hand side (and thus the right hand side) of the latter identity is an absolutely continuous function. Hence,

$$
u_{j}(t)=p_{j, j+1}^{-1}(t) \frac{d}{d t}\left(u_{j-1}(t)-u_{j-1}\left(t_{0}\right)-\sum_{k=1}^{j} \int_{t_{0}}^{t}\left(d \mathbf{p}_{j, k}\right) u_{k-1}(s)\right)
$$

as $j=1, \ldots, r-1$. Identity (19) holds true on the segment $[a, b]$. It follows from (19) that quasi-derivatives $u_{j}$ are determined uniquely by function $u=u_{0}$. We call function $u$ a solution to (17) if the system of functions $\widehat{u}$ solves 18 .

Let $W_{m}(t, \lambda)$ be an operator solution to (17) as $f=0, t_{0}=a_{0}$, satisfying the condition $W_{m}^{[j-1]}\left(a_{0}, \lambda\right)=\delta_{j m} E\left(\delta_{j m}\right.$ is the Kronecker delta, $\left.j, m=1, \ldots, r\right) ; \widehat{W}(t, \lambda)$ is the matrix with the entries $W_{m}^{[j-1]}(t, \lambda)$. Then th function $t \rightarrow \widehat{W}(t, \lambda)$ is a solution to equation (18) as $\breve{f}=0$.

Spaces $\mathfrak{H}=L_{2}(H, d \mathbf{m} ; a, b)$ and $L_{2}\left(\mathcal{H}, d \mathbf{m}_{1} ; a, b\right)$ coincide. Each function $\operatorname{col}\left(0, y_{2}, \ldots, y_{r-1}\right)$ with values in $H=\mathcal{H}^{r}$ is identified with zero in $\mathfrak{H}$. In the finite-dimensional case $Q_{-}=Q$, the maximal and minimal relations generated by system $(17)$ are defined as follows.

Maximal relation $L$ is the set of pairs $\{\tilde{u}, \tilde{f}\} \in \mathfrak{H} \times \mathfrak{H}$ such there exists a pair $\{u, f\}$ identified with $\{\tilde{u}, \tilde{f}\}$ in $\mathfrak{H} \times \mathfrak{H}$ and satisfying system (17) as $\lambda=0$. Minimal relation $L_{0}$ is the restriction of $L$ to the set of functions $u$ such that $\widehat{u}\left(a_{0}\right)=\widehat{u}\left(b_{0}\right)=0$, where $u$ is the solution to (17).

The boundary values are defined by formulae (15)

$$
\mathrm{Y}=\tilde{\delta}_{1}\{u, f\}=\widehat{u}\left(a_{0}\right), \quad \mathrm{Y}^{\prime}=\tilde{\delta}_{2}\{u, f\}=\widehat{u}\left(b_{0}\right)-\widehat{W}\left(b_{0}, 0\right) \widehat{u}\left(a_{0}\right)
$$

Then $\Phi_{\delta(\lambda)}=\widehat{W}\left(b_{0}, \lambda\right)-\widehat{W}\left(b_{0}, 0\right)$.

System of integral equations (17) satisfy the statement similar to Theorem 3 . We note that in the finite-dimensional case Conditions 1), 3), 5), 6) hold true immediately.

Remark 2. Let all the measures $\mathbf{p}_{j, k}$ be absolutely continuous, i.e., $\mathbf{p}_{j, k}(\Delta)=\int_{\Delta} p_{j, k}(t) d t$, $\left\|p_{j, k}(t)\right\| \in L_{1}(a, b)$, and $\mathbf{m}_{1}(\Delta)=\mu(\Delta) E$, where $\mu$ is the usual Lebesgue measure on $[a, b]$, i.e., $\mu([\alpha, \beta))=\beta-\alpha, \alpha, \beta \in \mathbb{R}, \alpha<\beta$ (as above, we let $\mu(\Delta)=0$ for each Borel set $\Delta$ such that $[a, b] \cap \Delta=\varnothing)$. Then $u^{[j]}$ are quasi-derivatives in the sense of [8], [9]. At that, $u^{[r]}=i^{-r} f$, where

$$
u^{[r]}=\left(u^{[r-1]}\right)^{\prime}-\sum_{k=1}^{r} p_{r, k}(t) u^{[k-1]} .
$$

\section{INTEGRAL EQUATIONS WITH IMPULSE ACTIONS}

In this section $H$ stands for a separable Hilbert space and $\mathbf{m}(\Delta)=\mu(\Delta) E$, where $\mu$ is the usual Lebesgue measure on $[a, b]$. In this case relation $L$ (and hence, $L_{0}$ ) is operator $Q_{-}=Q_{+}=$ $H$. Boundary values are defined by identities $(15)$, while operator $\Phi_{\delta(\lambda)}$ is introduced by identity (16). Moreover, for each $\tau \in\left[a_{0}, b_{0}\right]$, the operator $\{y, f\} \rightarrow y(\tau)$ maps continuously $L$ onto $H$. This is why the boundary values can be determined by the formulae $\mathrm{Y}=y\left(a_{0}\right), \mathrm{Y}^{\prime}=y\left(b_{0}\right)$. Then $\Phi_{\delta(\lambda)}=W\left(b_{0}, \lambda\right)$. Thus, in Theorem 3 and Corollary 4 , as $\Phi_{\delta(\lambda)}$ we can take the operator defined by identity (16) or operator $W\left(b_{0}, \lambda\right)$ (subject to the choice of SBV).

We note that in paper [16], in other way there were obtained statements similar to Theorem 3 and Corollary 4 for the differential operator generated by a strongly continuous family 
of evolution operators $\mathcal{U}(t, s)$ in a Banach space. These statements in [16] can be proven by a slight modification of the approach employed in the present work in view of Theorem 2 valid for Banach spaces (at that, as operator $\Phi_{\delta(\lambda)}=W\left(b_{0}, \lambda\right)$ we take $\left.e^{\lambda(b-a)} \mathcal{U}(b, a)\right)$.

We proceed to considering equation (1) with a multi-valued impulse action assuming that $\mathbf{m}(\Delta)=\mu(\Delta) E, t_{0}=a_{0}$, in (1).

We fix a point $t_{1} \in[a, b]$. We define a possible change of the solution at point $t_{1}$ as follows. We let

$$
\begin{array}{ll}
y(t)=W(t, 0) \tilde{c}_{1}-W(t, 0) i J \int_{a_{0}}^{t} U^{*}(s, 0) f(s) d s, & a_{0} \leqslant t \leqslant t_{1}, \\
y(t)=W(t, 0) W_{+}^{-1}\left(t_{1}, 0\right) \tilde{c}_{2}-W(t, 0) i J \int_{t_{1}}^{t} U^{*}(s, 0) f(s) d s, & t_{1}<t \leqslant b_{0},
\end{array}
$$

where $f \in \mathfrak{H}, \tilde{c}_{1}, \tilde{c}_{2} \in H, W_{+}\left(t_{1}, 0\right)=\lim _{t \rightarrow t_{1}+0} W(t, 0)$. Generally speaking, function $y$ has a jump at point $t_{1}$ because element $\tilde{c}_{2} \in H$ is chosen arbitrarily. We observe that $\tilde{c}_{1}=y\left(a_{0}\right)$, $\tilde{c}_{2}=\lim _{t \rightarrow t_{1}+0} y(t)$.

We define operator $\mathcal{L}$ as follows. We assume that domain $\mathcal{D}(\mathcal{L})$ of operator $\mathcal{L}$ consists of functions $y$ satisfying (20), (21) and we suppose that $\mathcal{L} y=f$. Operator $\mathcal{L}$ is closed.

In the definition of SBV we let $B_{1}=B_{2}=H \times H$ and we define boundary values by the identities

$$
\gamma_{1}\{y, f\}=\mathrm{Y}=\left\{y\left(a_{0}\right), y^{+}\left(t_{1}\right)\right\}, \quad \gamma_{2}\{y, f\}=\mathrm{Y}^{\prime}=\left\{y\left(t_{1}\right), y\left(b_{0}\right)\right\},
$$

where $y^{+}\left(t_{1}\right)=\lim _{t \rightarrow t_{1}+0} y(t)$. Lemma 2, Corollary 3 , and the continuous invertibility of operator $W(t, 0): H \rightarrow H$ yield that the quadruple $\left(H \times H, H \times H, \gamma_{1}, \gamma_{2}\right)$ is a SBV for operator $\mathcal{L}$. We let $\gamma\{y, f\}=\left\{\mathrm{Y}, \mathrm{Y}^{\prime}\right\}$.

Operator $\Phi_{\gamma}$ is defined by the identity

$$
\Phi_{\gamma}\left(\left\{\tilde{c}_{1}, \tilde{c}_{2}\right\}\right)=\left\{W\left(t_{1}, 0\right) \tilde{c}_{1}, W\left(b_{0}, 0\right) W_{+}^{-1}\left(t_{1}, 0\right) \tilde{c}_{2}\right\}, \quad\left\{\tilde{c}_{1}, \tilde{c}_{2}\right\} \in H \times H .
$$

Here we have taken into consideration that the function $t \rightarrow W(t, 0)$ is left continuous. In this case minimal operator $\mathcal{L}_{0}$ is introduced as the restriction of operator $\mathcal{L}$ to the set of functions $y \in \mathcal{D}(\mathcal{L})$ obeying $y\left(a_{0}\right)=y\left(b_{0}\right)=y\left(t_{1}\right)=y^{+}\left(t_{1}\right)=0$.

Let $\theta$ be a linear relation, $\theta \subset(H \times H) \times(H \times H), \mathcal{L}_{\theta}$ be an operator such that $\mathcal{L}_{0} \subset \mathcal{L}_{\theta} \subset \mathcal{L}$ and $\gamma \mathcal{L}_{\theta}=\theta$. Operator $\mathcal{L}_{\theta}$ satisfy the statements similar to Theorem 3 and Corollary 4 .

We consider the important particular case when relation $\theta$ is determined by two relations $\theta_{12}$ and $\theta_{21}$ consisting of the pairs of boundary values at the discontinuity point $t_{1}$ and the pairs of boundary values at the end-points $a_{0}, b_{0}$, respectively. We denote by $H_{1}, H_{2}$ the first and second copy of space $H$ in the Cartesian product $H \times H$ and we assume that the relation $\theta \subset\left(H_{1} \times H_{2}\right) \times\left(H_{1} \times H_{2}\right)$ consists of pairs

$$
\left\{\operatorname{col}\left(x_{1}, x_{2}\right), \operatorname{col}\left(x_{12}, x_{21}\right)\right\},
$$

where $\left\{x_{2}, x_{12}\right\} \in \theta_{12} \subset H_{2} \times H_{1},\left\{x_{1}, x_{21}\right\} \in \theta_{21} \subset H_{1} \times H_{2}$ (hereinafter it is convenient to denote a pair $\left\{z_{1}, z_{2}\right\} \in H_{1} \times H_{2}$ as the column $\operatorname{col}\left(z_{1}, z_{2}\right)$ to track the analogy with the operators defined by matrices). Thus, the domain of operator $\mathcal{L}_{\theta}$ consists of functions $y$ defined by (20), 21) and satisfying the boundary conditions

$$
\left\{y\left(a_{0}\right), y\left(b_{0}\right)\right\} \in \theta_{21}, \quad\left\{y^{+}\left(t_{1}\right), y\left(t_{1}\right)\right\} \in \theta_{12} .
$$

We note that relation $\theta$ is closed if and only if relations $\theta_{12}$ and $\theta_{21}$ closed. In what follows we assume that relation $\theta$ is closed.

For the sake of shortening the notations we denote $\omega_{1}=W\left(t_{1}, 0\right), \omega_{2}=W\left(b_{0}, 0\right) W_{+}^{-1}\left(t_{1}, 0\right)$. Let $\omega: H_{1} \times H_{2} \rightarrow H_{1} \times H_{2}$ be the operator defined by the identity $\omega\left\{x_{1}, x_{2}\right\}=\left\{\omega_{1} x_{1}, \omega_{2} x_{2}\right\}$, 
where $x_{1} \in H_{1}=H, x_{2} \in H_{2}=H$. It follows from (22), (23) that the relation $\theta-\Phi_{\gamma}$ consists of pairs

$$
\left\{\operatorname{col}\left(x_{1}, x_{2}\right), \operatorname{col}\left(-\omega_{1} x_{1}+x_{12}, x_{21}-\omega_{2} x_{2}\right)\right\},
$$

where $\left\{x_{2}, x_{12}\right\} \in \theta_{12},\left\{x_{1}, x_{21}\right\} \in \theta_{21}$.

Operator $\omega$ is a continuous one-to-one mapping of $H_{1} \times H_{2}$ onto $H_{1} \times H_{2}$. This is why the relations $\theta-\Phi_{\gamma}$ and $\zeta=\omega^{-1}\left(\theta-\Phi_{\gamma}\right)$ are simultaneously either in state or not in state $k$ $(1 \leqslant k \leqslant 8)$. We denote $\zeta_{12}=\omega_{1}^{-1} \theta_{12}, \zeta_{21}=\omega_{2}^{-1} \theta_{21}$. It follows from 24 that relation $\zeta$ consists of pair

$$
\left\{\operatorname{col}\left(g_{1}, g_{2}\right), \operatorname{col}\left(-g_{1}+g_{12}, g_{21}-g_{2}\right)\right\},
$$

where $\left\{g_{2}, g_{12}\right\} \in \zeta_{12},\left\{g_{1}, g_{21}\right\} \in \zeta_{21}$.

Lemma 4. The following statements hold true: a) $\operatorname{dim} \operatorname{ker} \zeta<\infty$ if and only if $\operatorname{dim} \operatorname{ker}\left(\zeta_{12} \zeta_{21}-E\right)<\infty$ and $\left.\operatorname{dim} \operatorname{ker}\left(\zeta_{21} \zeta_{12}-E\right)<\infty ; b\right) \operatorname{dim} \operatorname{ker} \zeta=0$ if and only if $\operatorname{dim} \operatorname{ker}\left(\zeta_{12} \zeta_{21}-E\right)=0$ and $\operatorname{dim} \operatorname{ker}\left(\zeta_{21} \zeta_{12}-E\right)=0$.

Proof. Let $\operatorname{col}\left(g_{1}, g_{2}\right) \in \operatorname{ker} \zeta$. It follows 25 that there exist elements $g_{12}, g_{21} \in H$ such that $\left\{g_{2}, g_{12}\right\} \in \zeta_{12},\left\{g_{1}, g_{21}\right\} \in \zeta_{21}$ and $g_{1}=g_{12}, g_{2}=g_{21}$. It yields that $\left\{g_{1}, g_{12}\right\} \in \zeta_{12} \zeta_{21}$ and $g_{1} \in \operatorname{ker}\left(\zeta_{12} \zeta_{21}-E\right)$. In the same way we obtain $\left\{g_{2}, g_{21}\right\} \in \zeta_{21} \zeta_{12}$ and $g_{2} \in \operatorname{ker}\left(\zeta_{21} \zeta_{12}-E\right)$.

On the other hand, if $g_{1} \in \operatorname{ker}\left(\zeta_{12} \zeta_{21}-E\right)$, there exist elements $g_{21}$ and $g_{12}$ such that $\left\{g_{1}, g_{21}\right\} \in \zeta_{21},\left\{g_{21}, g_{12}\right\} \in \zeta_{12}$ and $g_{12}=g_{1}$. Together with 25) it implies $\operatorname{col}\left(g_{1}, g_{21}\right) \in \operatorname{ker} \zeta$. In the same way we obtain that if $g_{2} \in \operatorname{ker}\left(\zeta_{21} \zeta_{12}-E\right)$, there exists an element $g_{12}$ with the property $\operatorname{col}\left(g_{12}, g_{2}\right) \in \operatorname{ker} \zeta$. The above arguments imply the statement of the lemma. The proof is complete.

We denote $Z_{1}=\mathcal{R}\left(\zeta_{12} \zeta_{21}-E\right), Z_{2}=\mathcal{R}\left(\zeta_{21} \zeta_{12}-E\right)$.

Lemma 5. Relation $\zeta$ is surjective if and only if the relations $\zeta_{12} \zeta_{21}-E$ and $\zeta_{21} \zeta_{12}-E$ are surjective.

Proof. Suppose that relation $\zeta$ is surjective. It follows from (25) that for each $z_{1}, z_{2} \in H$ there exist pairs $\left\{g_{2}, g_{12}\right\} \in \zeta_{12},\left\{g_{1}, g_{21}\right\} \in \zeta_{21}$ such that $-g_{1}+g_{12}=z_{1}, g_{21}-g_{2}=z_{2}$. We let $z_{2}=0$. Then $g_{2}=g_{21}$. This is why $\left\{g_{1}, g_{12}\right\} \in \zeta_{12} \zeta_{21}$ and $z_{1} \in Z_{1}=\mathcal{R}\left(\zeta_{12} \zeta_{21}-E\right)$. By the arbitrariness of $z_{1}$ we obtain $Z_{1}=H$. In the same way we prove that $Z_{2}=\mathcal{R}\left(\zeta_{21} \zeta_{12}-E\right)=H$. Thus, relations $\zeta_{12} \zeta_{21}-E, \zeta_{21} \zeta_{12}-E$ are surjective.

Let us prove the opposite statement. We have $z_{2} \in Z_{2}$ if and only if there exists an element $g_{2}$ such that $\left\{g_{2}, z_{2}\right\} \in \zeta_{21} \zeta_{12}-E$. It is equivalent to the existence of elements $g_{12}, g_{21}$ with the properties

$$
\left\{g_{2}, g_{12}\right\} \in \zeta_{12}, \quad\left\{g_{12}, g_{21}\right\} \in \zeta_{21}, \quad g_{21}-g_{2}=z_{2} .
$$

In the same way, $z_{1} \in Z_{1}$ if and only if there exist elements $g_{1}, g_{12}^{\prime}, g_{21}^{\prime}$ with the properties

$$
\left\{g_{1}, z_{1}\right\} \in \zeta_{12} \zeta_{21}-E, \quad\left\{g_{1}, g_{21}^{\prime}\right\} \in \zeta_{21}, \quad\left\{g_{21}^{\prime}, g_{12}^{\prime}\right\} \in \zeta_{12}, \quad g_{12}^{\prime}-g_{1}=z_{1} .
$$

By (26), (27) we obtain that $\left\{g_{2}+g_{21}^{\prime}, g_{12}+g_{12}^{\prime}\right\} \in \zeta_{12},\left\{g_{12}+g_{1}, g_{21}+g_{21}^{\prime}\right\} \in \zeta_{21}$. Together with (25) it yields $\left\{\operatorname{col}\left(g_{12}+g_{1}, g_{2}+g_{21}^{\prime}\right), \operatorname{col}\left(g_{12}^{\prime}-g_{1}, g_{21}-g_{2}\right)\right\} \in \zeta$. Identities (26), (27) imply $\left\{\operatorname{col}\left(g_{12}+g_{1}, g_{2}+g_{21}^{\prime}\right), \operatorname{col}\left(z_{1}, z_{2}\right)\right\} \in \zeta$. Hence, $\operatorname{col}\left(z_{1}, z_{2}\right) \in \mathcal{R}(\zeta)$.

Thus, if the relations $\zeta_{12} \zeta_{21}-E$ and $\zeta_{21} \zeta_{12}-E$ are surjective, then relation $\zeta$ is surjective. The proof is complete.

Remark 3. In the proof of the second part of Lemma 5 we have in fact established the following statement: if $z_{1} \in Z_{1}, z_{2} \in Z_{2}$, then $\operatorname{col}\left(z_{1}, z_{2}\right) \in \mathcal{R}(\zeta)$. 
Theorem 4. Operator $\mathcal{L}_{\theta}$ is continuously invertible if and only if the relations

$$
W^{-1}\left(t_{1}, 0\right) \theta_{12} W_{+}\left(t_{1}, 0\right) W^{-1}\left(b_{0}, 0\right) \theta_{21}-E, \quad W_{+}\left(t_{1}, 0\right) W^{-1}\left(b_{0}, 0\right) \theta_{21} W^{-1}\left(t_{1}, 0\right) \theta_{12}-E
$$

are continuously invertible.

Proof. Relations (28) are equal to $\zeta_{12} \zeta_{21}-E$ and $\zeta_{21} \zeta_{12}-E$, respectively. It was established above that $\mathcal{L}_{\theta}, \theta-\Phi_{\gamma}, \zeta$ are simultaneously in state $k(1 \leqslant k \leqslant 8)$. Now the desired statement follow from Lemmata 4,5 .

Lemma 6. If range $\mathcal{R}(\zeta)$ is closed and has a finite codimension, then the ranges $Z_{1}=$ $\mathcal{R}\left(\zeta_{12} \zeta_{21}-E\right)$ and $Z_{2}=\mathcal{R}\left(\zeta_{21} \zeta_{12}-E\right)$ have a finite codimension. If $Z_{1}, Z_{2}$ have a finite codimension, the same is true for $\mathcal{R}(\zeta)$.

Proof. Suppose that $\mathcal{R}(\zeta)$ is closed and has a finite codimension. Then $\mathcal{R}(\zeta) \cap(H \times\{0\})$ has a finite codimension. Let $\operatorname{col}\left(z_{1}, 0\right) \in \mathcal{R}(\zeta) \cap(H \times\{0\})$. As in the proof of the first part of Lemma 5 we obtain $z_{1} \in Z_{1}$. It yields that $Z_{1}$ has a finite codimension. The required statement on $Z_{2}$ can be proven in the same way.

Suppose that $Z_{1}$ and $Z_{2}$ have finite codimension $z_{1} \in Z_{1}, z_{2} \in Z_{2}$. By Remark 3 we obtain $\operatorname{col}\left(z_{1}, z_{2}\right) \in \mathcal{R}(\zeta)$. Hence, $\mathcal{R}(\zeta)$ has a finite codimension. The proof is complete.

Theorem 5. Operator $\mathcal{L}_{\theta}$ is Fredholm if and only if relations (28) are Fredholm.

Proof. Operator $\mathcal{L}_{\theta}$ and relations $\theta-\Phi_{\gamma}, \zeta$ are simultaneously either Fredholm or not. Suppose that relation $\zeta$ is Fredholm. Then range $\mathcal{R}(\zeta)$ is closed. Let us prove the same for $Z_{1}=$ $\mathcal{R}\left(\zeta_{12} \zeta_{21}-E\right)$. Suppose that $z_{1, n} \in Z_{1}$ and sequence $\left\{z_{1, n}\right\}$ converges to $z$. It follows from Remark 3 that $\left.\operatorname{col}\left(z_{1, n}, 0\right)\right\} \in \mathcal{R}(\zeta)$. Closeness of $\mathcal{R}(\zeta)$ implies $\operatorname{col}(z, 0) \in \mathcal{R}(\zeta)$. By the proof of the first part of Lemma 5 we obtain that $z \in Z_{1}$. The closeness of $Z_{2}=\mathcal{R}\left(\zeta_{12} \zeta_{21}-E\right)$ can be established in the same way. Now the Fredholm property for relations $(28)$ follow from Lemmata 4, 6.

Vice versa, let relations $(28)$ be Fredholm. These relations are equal to $\zeta_{12} \zeta_{21}-E$ and $\zeta_{21} \zeta_{12}-E$, respectively. Therefore, $Z_{1}, Z_{2}$ closed. This set $Z_{1} \times Z_{2}$ is closed. It has a finite codimension in $H \times H$, since by the assumption $Z_{1}, Z_{2}$ have a finite codimension. Therefore, there exists a linear manifold $M \subset H \times H$ such that $\operatorname{dim} M<\infty,\left(Z_{1} \times Z_{2}\right) \cap M=\{0,0\}$ and $H \times H=\left(Z_{1} \times Z_{2}\right) \dot{+} M$. By Remark 3 we obtain $Z_{1} \times Z_{2} \subset \mathcal{R}(\zeta)$. Hence, $\mathcal{R}(\zeta)=$ $\left(Z_{1} \times Z_{2}\right) \dot{+}(M \cap \mathcal{R}(\zeta))$. It follows from [17, Ch. 1, Prop. 3.3] that $\mathcal{R}(\zeta)$ is closed. By applying Lemmata 4,6 we complete the proof.

\section{BIBLIOGRAPHY}

1. Yu.V. Pokornyi, M.B. Zvereva, S.A. Shabrov. Sturm-Liouville oscillation theory for impulsive problems // Uspekhi Matem. Nauk. 63:1, 111-154 (2008). [Russ. Math. Surv. 63:1, 109-153 (2008).]

2. A.M. Savchuk, A.A. Shkalikov. Sturm-liouville operators with singular potentials // Matem. Zametki. 66:6, 897-912 (1999). [Math. Notes. 66:6, 741-753 (1999).]

3. V.M. Bruk. Invertible linear relations generated by an integral equation with Nevanlinna measure // Izv. VUZov. Matem. 2, 16-29 (2013). [Russ. Math. 57:2, 13-24 (2013).]

4. A.G. Baskakov. Spectral analysis of differential operators with unbounded operator-valued coeffcients, difference relations and semigroups of difference relations // Izv. RAN. Ser. Matem. 73:2, 3-68 (2009). [Izvestiya: Mathematics. 73:2, 215-278 (2009).]

5. A.G. Baskakov. Analysis of linear differential equations by methods of the spectral theory of difference operators and linear relations // Uspekhi Matem. Nauk. 68:1, 77-128 (2013). [Russ. Math. Surv. 68:1, 69-116 (2013).]

6. V.M. Bruk. On invertible restrictions of closed operators in Banach spaces // Funkts. Anal. 28, 17-22 (1988). (in Russian). 
7. V.M. Bruk. On linear relations generated by Nonnegative operator function and degenerate elliptic differential-operator expression // Zh. Mat. Fiz. Anal. Geom. 5:2, 123-144 (2009).

8. D. Shin. Quasi-differential operators in Hilbert space // Matem. Sbornik. 13:1, 39-70 (1943). (in Russian).

9. A. Zettl. Formally self-adjoint quasi-differential operators // Rocky Mountain J. Math. 5:3, 453474 (1975).

10. A.M. Samojlenko, N.A. Perestyuk. Differential equations with impulse action. Vischa Schkola, Kiev (1987).

11. V.B. Didenko. On the continuous invertibility and the Fredholm property of differential operators with multi-valued impulse effects // Izv. RAN. Ser. Matem. 77:1, 5-22 (2013). [Izvestiya: Math. 77:1, 3-19 (2013).]

12. F.S. Rofe-Beketov. Selfadjoint extensions of differential operators in a space of vector functions // Dokl. Akad. Nauk SSSR. 184:5, 1034-1037 (1969). [Soviet. Math. Dokl. 10:1, 188-192 (1969).]

13. Yu.M. Berezanski. Expansions in Eigenfunctions of Selfadjoint Operators. Naukova Dumka, Kiev (1965). [Amer. Math. Soc., Providence, RI, (1968).]

14. V.M. Bruk. On the characteristic operator of an integral equation with a nevanlinna measure in the infinite-dimensional case // Zh. Mat. Fiz. Anal. Geom. 10:2, 163-188 (2014). (in Russian.)

15. V.M. Bruk. On invertible restrictions of relations generated by a differential expression and by a Nonnegative operator function // Matem. Zametki. 82:5, 652-664 (2007). [Math. Notes. 82:5, 583-595.]

16. V.B. Didenko. On the spectral properties of differential operators with unbounded operator coefficients determined by a linear relation // Matem. Zametki. 89:2, 226-240 (2011). [Math. Notes. 89:2, 224-237 (2011).]

17. H. Schaefer. Topological Vector Spaces. The Macmillan Company, London, (1966).

Vladislav Moiseevich Bruk,

Saratov State Technical University,

Politechnicheskaya str., 77,

410054, Saratov, Russia

E-mail: vladislavbruk@mail.ru 